\title{
Temporal quadratic solitons and their interaction with dispersive waves in lithium niobate nanowaveguides
}

\author{
William R. Rowe ${ }^{*},{ }^{*}$ Dmitry V. Skryabin $\odot$, and Andrey V. Gorbach ${ }^{\dagger}{ }^{\dagger}$ \\ Centre for Photonics and Photonic Materials, Department of Physics, University of Bath, Bath BA2 7AY, United Kingdom
}

(Received 2 August 2019; published 3 December 2019)

\begin{abstract}
We present a model of soliton propagation in waveguides with quadratic nonlinearity. Criteria for solitons to exist in such waveguides are developed and two example nanowaveguide structures are simulated as proof of concept. Interactions between quadratic solitons and dispersive waves are analyzed, giving predictions closely matching soliton propagation simulations. The example structures are found to support five different regimes of soliton and quasisoliton existence. Pulse propagation in these example waveguides has been simulated confirming the possibility of soliton generation at experimentally accessible powers. Simulations of multisoliton generation, Cherenkov radiation, and quasisolitons with opposite signs of dispersion in the fundamental and second harmonic are also presented here.
\end{abstract}

DOI: 10.1103/PhysRevResearch.1.033146

\section{INTRODUCTION}

Temporal solitons are an important class of solitary waves well known in the context of nonlinear optics [1]. Perhaps the most studied type of temporal solitons are cubic solitons, whereby self-action due to cubic $\left(\chi^{(3)}\right.$ or Kerr) nonlinearity (typically self-focusing) balances dispersion, hence leading to formation of stable self-localized pulses of light propagating in optical waveguides or fibers [2]. Effective self-action can also occur in quadratic $\left(\chi^{(2)}\right)$ nonlinear media due to cascaded interactions between the fundamental frequency (FF) and second harmonic (SH) [3]. In the limit of a large phase mismatch between FF and $\mathrm{SH}$, the so-called cascading limit $[3,4]$, the system of coupled equations for FF and SH components can be reduced to an effective Kerr-type system. Notably, the sign of such effective Kerr interaction is controlled by the sign of the phase mismatch. This makes the parameter space of existence of such cascaded $\chi^{(2)}$ temporal solitons considerably wider than in native $\chi^{(3)}$ systems [4]. However, existence of quadratic temporal solitons away from the cascading limit requires a more complicated balance between nonlinear interaction, dispersion, and walk-off due to the mismatch of group velocities of the two copropagating FF and SH pulses.

Interactions between solitons and small-amplitude dispersive waves is a well-known generic mechanism of frequency conversion in Kerr media, and plays a crucial role in supercontinuum generation in optical fibers [5]. The corresponding

\footnotetext{
*w.r.rowe@bath.ac.uk

†.gorbach@bath.ac.uk
}

Published by the American Physical Society under the terms of the Creative Commons Attribution 4.0 International license. Further distribution of this work must maintain attribution to the author(s) and the published article's title, journal citation, and DOI. theory is developed for Kerr solitons [6], and recently was extended to cases including $\chi^{(2)}$ as a perturbation to Kerr solitons [7] and $\chi^{(2)}$ solitons in the cascaded limit [8,9]. Importantly this previous work does not include any predictions for dispersive waves emitted from the SH component in $\chi^{(2)}$ solitons. We also note the existence of embedded solitons which do not emit radiation despite their spectrum overlapping with dispersive waves [10].

Recent advancements in the fabrication of lithium niobate (LN) nanowaveguides [11] has reignited interest in this well-characterized $\chi^{(2)}$ material [12]. The small mode size in these nanowaveguides enhances nonlinearity [11], therefore reducing required peak powers to achieve efficient nonlinear interactions. Their strong guidance also provides geometrically tuneable dispersion allowing direct phase matching between modes [13,14], as well as considerable reduction of group velocity mismatch between $\mathrm{FF}$ and $\mathrm{SH}$ modes within wide frequency ranges [15]. Continued research has seen the loss in these structures fall as low as $0.027 \mathrm{~dB} / \mathrm{cm}$ [16], further improving the prospects of these structures for practical application. These LN nanowaveguides therefore provide novel opportunity for $\chi^{(2)}$ soliton research.

In this work a model of temporal quadratic solitons in $\chi^{(2)}$ waveguides is developed. Criteria for the existence of localized soliton solutions based on waveguide parameters are derived. The model is then extended to include the interaction of the soliton with dispersive waves. Two examples of nanowaveguide structures are simulated and found to support soliton and quasisoliton existence in the normal dispersion regime, at experimentally attainable powers. Simulations of pulse propagation in the example nanowaveguide structures are presented and validate predictions of soliton existence and the frequency of resonant radiation. This work develops theory of important frequency conversion phenomena with potential to enhance understanding of broadband supercontinuum generation in $\chi^{(2)}$ waveguides. 


\section{MODEL}

We consider a generic $\chi^{(2)}$ waveguide with a fixed cross section in the Cartesian $x-y$ plane and invariant along $z$, the propagation direction. We assume two pulsed light fields are excited in different modes of the waveguide at a frequency $\omega_{f}$ (fundamental field, FF) and its second harmonic (SH) $\omega_{s}=$ $2 \omega_{f}$ with envelope functions $U_{f}(z, t)$ and $U_{s}(z, t)$ respectively. The propagation constant $\beta_{m}(\omega)$ for waveguide mode $m$ is related to the effective refractive index of that mode, $n_{\mathrm{eff}, m}$, by

$$
\beta_{m}(\omega)=\omega n_{\mathrm{eff}, m}(\omega) / c,
$$

where $m=f$ and $m=s$ label the waveguide mode chosen for the FF and SH respectively. The $j$ th derivative of $\beta_{m}$ with respect to frequency is therefore

$$
\beta_{m j}=\frac{d^{j} \beta_{m}}{d \omega^{j}}=\frac{1}{c}\left[j \frac{d^{j-1} n_{\mathrm{eff}, m}}{d \omega^{j-1}}+\omega \frac{d^{j} n_{\mathrm{eff}, m}}{d \omega^{j}}\right] .
$$

The evolution of the field envelopes is described by

$$
\begin{gathered}
i \partial_{\xi} U_{f}=-D_{f}\left(i \partial_{\tau}\right) U_{f}-U_{s} U_{f}^{*} e^{i \kappa \xi}, \\
i \partial_{\xi} U_{s}=-D_{s}\left(i \partial_{\tau}\right) U_{s}-\frac{U_{f}^{2}}{2} e^{-i \kappa \xi},
\end{gathered}
$$

where $t$ has been shifted to move with the FF pulse group velocity (GV) $v_{f}=\left.\beta_{f 1}^{-1}\right|_{\omega_{f}}$ and normalized by the approximate pulse duration $t_{0}$, giving $\tau=\left(t-z / v_{f}\right) / t_{0}$. $z$ has been normalized by the dispersion length $z_{d}=2 t_{0}^{2} /\left|\beta_{f 2}\right|$ to give $\xi=z / z_{d}$. The phase mismatch $\kappa=\Delta \beta z_{d}$, where $\Delta \beta=$ $\beta_{s}\left(2 \omega_{f}\right)-2 \beta_{f}\left(\omega_{f}\right)$ (note that in the literature $\kappa$ is often defined with the opposite sign). From the definition of $\beta_{m}$ in Eq. (1) it follows that

$$
\kappa=2 z_{d} \omega_{f}\left[n_{\mathrm{eff}, s}\left(2 \omega_{f}\right)-n_{\mathrm{eff}, f}\left(\omega_{f}\right)\right] .
$$

The dispersion operators are found by taking a Taylor series,

$$
\begin{aligned}
& D_{f}\left(i \partial_{\tau}\right)=-\sum_{j=2}^{+\infty} r_{j}\left[i \partial_{\tau}\right]^{j}, \\
& D_{s}\left(i \partial_{\tau}\right)=+s_{1}\left[i \partial_{\tau}\right]-\sum_{j=2}^{+\infty} s_{j}\left[i \partial_{\tau}\right]^{j} .
\end{aligned}
$$

The walk-off parameter $s_{1}=z_{d} / z_{w}$ where $z_{w}=t_{0} /\left(v_{s}^{-1}-\right.$ $v_{f}^{-1}$ ) is the walk-off length where the SH GV, $v_{s}=\left.\beta_{s 1}^{-1}\right|_{2 \omega_{f}}$. The remaining dispersion coefficients are

$$
r_{j}=-\frac{z_{d}}{t_{0}^{j} j !} \beta_{f j}, \quad s_{j}=-\frac{z_{d}}{t_{0}^{j} j !} \beta_{s j},
$$

for integers $j \geqslant 2$. It should be noted that $r_{2}= \pm 1$. The dispersion of each mode at frequency detuning $\delta=(\omega-$ $\left.\omega_{f}\right) t_{0}=\left(\omega-\omega_{s}\right) t_{0}$ is therefore given by

$$
D_{f}(\delta)=-\sum_{j=2}^{+\infty} r_{j} \delta^{j}, \quad D_{s}(\delta)=s_{1} \delta-\sum_{j=2}^{+\infty} s_{j} \delta^{j} .
$$

Fields are scaled such that $U_{f}=\sqrt{2} \rho_{2} z_{d} A_{f}$ and $U_{s}=$ $\rho_{2} z_{d} A_{s}$, where $\left|A_{f, s}\right|^{2}$ are intensities in watts. The effective nonlinear coefficient [14]

$$
\rho_{2}=\frac{\varepsilon_{0} \omega_{f}}{4 \sqrt{N_{s} N_{f}}} \iint \vec{e}_{s}\left[\hat{\chi}_{2}: \vec{e}_{f}^{2}\right] d A_{n},
$$

where $\vec{e}_{f, s}$ are the electric field profiles of the chosen FF and $\mathrm{SH}$ waveguide modes respectively. $\hat{\chi}_{2}$ is the second-order nonlinear tensor of the $\chi^{(2)}$ material in the waveguide and $A_{n}$ is the cross section of the $\chi^{(2)}$ material, over which the integral is performed. The normalization factors, $N_{m}=[1 / 4] \iint\left[\vec{e}_{m} \times\right.$ $\left.\vec{h}_{m}^{*}\right]+\left[\vec{e}_{m}^{*} \times \vec{h}_{m}\right] d A_{w}$, where $\vec{h}_{m}$ is the magnetic field profile for the mode $m$ and $A_{w}$ is the cross section of the whole waveguide, over which the integral is performed (not only the $\chi^{(2)}$ material).

\section{SOLITON THEORY}

\section{A. Tail analysis}

We first consider soliton solutions of the system in Eq. (3) with second-order dispersion only such that

$$
\begin{aligned}
& D_{f}(\delta)=-r_{2} \delta^{2}, \\
& D_{s}(\delta)=s_{1} \delta-s_{2} \delta^{2} .
\end{aligned}
$$

Solitons are sought in the form of localized pulses copropagating with a common group velocity $v$ :

$$
\begin{aligned}
U_{f} & =W_{f}(\eta) e^{i \mu \xi}, \\
U_{s} & =W_{s}(\eta) e^{i[2 \mu-\kappa] \xi},
\end{aligned}
$$

where $W_{f}$ and $W_{s}$ are the soliton field profiles. The transverse coordinate, $\eta=\tau-v \xi$, moves with the soliton velocity $v$, and $\mu$ is the nonlinear correction to propagation constant. $\mu$ and $\nu$ are the soliton family parameters. Substituting into Eq. (3) and requiring a nondispersive soliton solution $\left(\partial_{\xi} W_{f}=\right.$ $\left.\partial_{\xi} W_{s}=0\right)$ gives

$$
\begin{aligned}
{\left[-i v \partial_{\eta}+r_{2} \partial_{\eta}^{2}-\mu\right] W_{f}+W_{s} W_{f}^{*} } & =0, \\
{\left[i\left[s_{1}-\nu\right] \partial_{\eta}+s_{2} \partial_{\eta}^{2}-2 \mu+\kappa\right] W_{s}+\frac{W_{f}^{2}}{2} } & =0 .
\end{aligned}
$$

While exact solutions are known for these coupled equations under specific conditions [17], we work below with a generic family of fundamental solitons found numerically [4]. For large phase mismatch $\kappa$, neglecting SH dispersion $s_{2}$, these coupled equations simplify to the nonlinear Schrödinger (NLS) equation. With the appropriate combination of FF dispersion and phase-mismatch signs, such that $r_{2} \kappa<0$, bright soliton solutions are known to have the form [4]

$$
\begin{aligned}
& W_{f}\left(\eta-s_{1} \xi\right) \approx \pm 2 \mu \sqrt{\alpha} \operatorname{sech}\left(\sqrt{|\mu|}\left[\eta-s_{1} \xi\right]\right), \\
& W_{s}\left(\eta-s_{1} \xi\right) \approx 2 \mu \operatorname{sech}^{2}\left(\sqrt{|\mu|}\left[\eta-s_{1} \xi\right]\right),
\end{aligned}
$$

for $r_{2}= \pm 1$ where $\alpha=[2 \mu-\kappa] / \mu \gg 1$. In this limit the FF component is much greater than the $\mathrm{SH}$ and the soliton peak power increases with $|\mu|$.

Requiring that any soliton solution must be localized gives the opportunity for further analysis. To meet this requirement both frequency components of the soliton must be exponentially decaying far from the center. This can be enforced by setting the form of each soliton profile, $W_{f}=V_{f} e^{-\theta_{f}|\eta|}$ and 
$W_{s}=V_{s} e^{-\theta_{s}|\eta|}$, for $|\eta| \gg 1$. Operating far from the center of the soliton, $V_{f, s}$ are some small constant amplitudes, therefore Eq. (11) can be linearized. Then requiring that $\theta_{f}$ and $\theta_{s}$ have positive real parts ensures exponential decay and yields conditions for localization of the soliton as

$$
\begin{gathered}
4 r_{2} \mu>v^{2}, \\
4 s_{2}[2 \mu-\kappa]>\left[v-s_{1}\right]^{2} .
\end{gathered}
$$

While these conditions are required for exponential tails they are not sufficient for soliton existence. Another required condition is the existence of a constant amplitude (CA) solution that acts as the center of the homoclinic orbit corresponding to the soliton solution. To derive a condition for the existence of a CA solution we look at CA solution itself, found by others [18] to be

$$
\bar{U}_{f}= \pm[2 \mu\{2 \mu-\kappa\}]^{\frac{1}{2}}, \quad \bar{U}_{s}=\mu,
$$

where $U_{f}=\bar{U}_{f} e^{i \mu \xi}$ and $U_{s}=\bar{U}_{s} e^{i[2 \mu-\kappa] \xi}$. As the system [Eq. (3)] is invariant under the transformation

$$
\left(U_{f}, U_{s}\right) \rightarrow\left(U_{f} e^{i \phi}, U_{s} e^{2 i \phi}\right), \quad \forall \phi,
$$

both $U_{f}$ and $U_{s}$ can be chosen to be real (at $\xi=0$ ), and therefore have a fixed phase difference of either 0 or $\pi$. So the CA solution in Eq. (15) is only valid when it satisfies this fixed phase difference. As $\mu$ is real, $\bar{U}_{s}$ will always be real. For $\bar{U}_{f}$ to be real, however,

$$
\mu[2 \mu-\kappa]>0
$$

is required, giving our condition for the existence of a $\mathrm{CA}$ solution.

The conditions for localization and CA solution existence provide the criteria for localized soliton existence. Graphical representations of these criteria are given in Fig. 1 for various waveguide parameters. Each condition on soliton existence is represented by a shaded region in these plots and solitons may exist where all three regions overlap. The five examples given in Figs. 1(a)-1(e) represent distinct regimes of soliton existence. In these examples $r_{2}=-1$ has been chosen setting normal dispersion in the FF, which is the case for the rest of this work. With normal dispersion in the FF set, solutions in Eq. (12) are valid when $\kappa$ is large and positive.

Figures 1(a)-1(c) show regimes in which the phase mismatch $\kappa$ is positive. Conversely Figs. 1(d) and 1(e) show regimes of $\kappa<0$. In this text these regimes will be referred to by their figure labels [i.e., the regime in Fig. 1(a) is regime $\mathrm{A}]$.

Regimes A, B, and D [shown in Figs. 1(a), 1(b), and 1(d), respectively] have areas where all three shaded regions overlap. These regimes are therefore expected to support soliton existence. The difference between these regimes is the range of $|\mu|$ for which solitons are predicted. In regime A solitons are predicted for all negative $\mu$ whereas in regime B solitons are only expected to be possible when $|\mu|$ exceeds a certain threshold value. The key difference between regimes A and B is the shift of the $\mathrm{SH}$ localization condition [Eq. (14)] due to $s_{1}$. The overlap of FF and SH localization conditions down to $|\mu|=0$ is determined by the condition

$$
\kappa>-s_{1}^{2} / 4 s_{2} \text {. }
$$
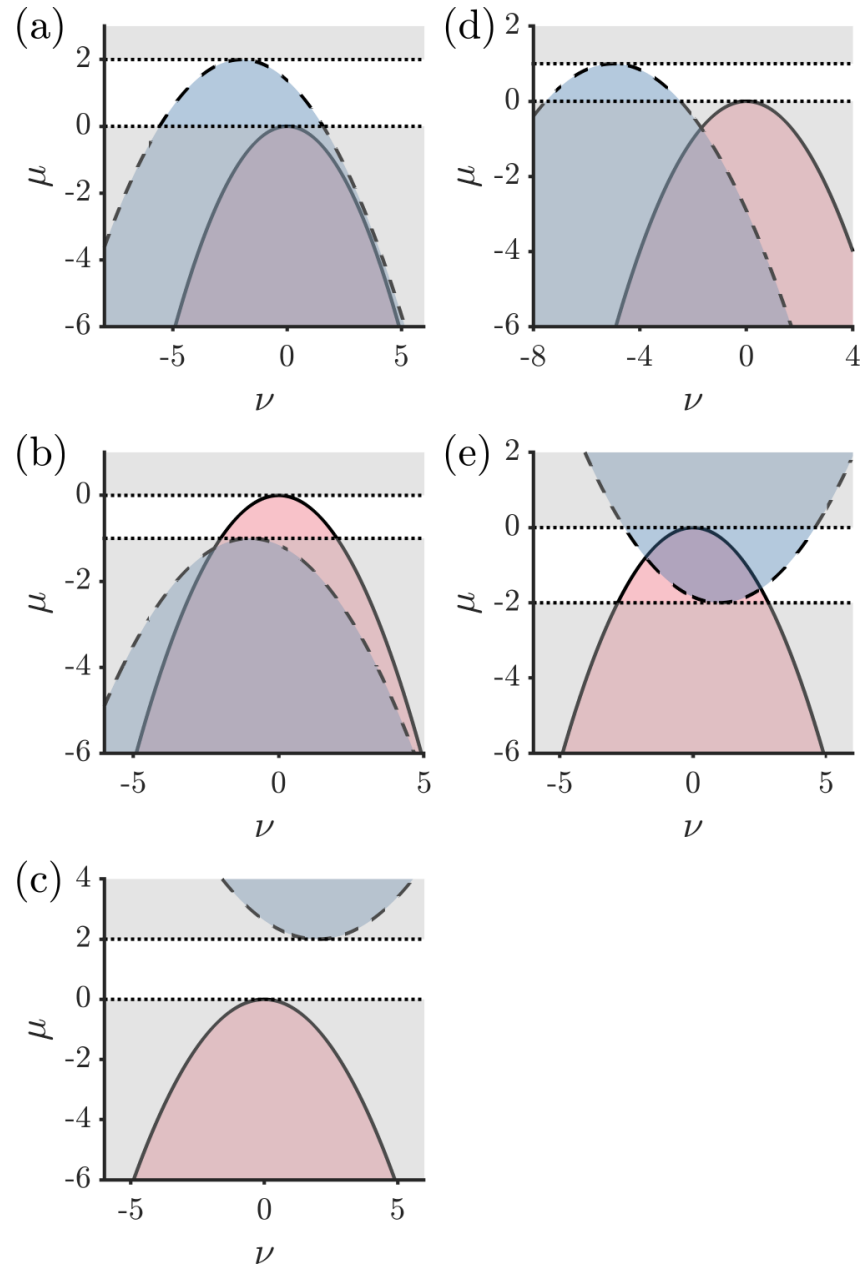

FIG. 1. Localization conditions, Eqs. (13) and (14), against soliton parameters $\mu$ and $v$. Conditions in Eqs. (13) and (14) are marked as shaded regions (in red and blue, respectively) and bounded by solid and dashed lines, respectively. Grey shaded regions bounded by dotted lines mark where constant amplitude solutions exist according to the condition in Eq. (17). Parameter $r_{2}=-1$ throughout, and (a) $s_{2}=-0.8, \kappa=4, s_{1}=-2$. (b) $s_{2}=-0.8, \kappa=2, s_{1}=$ -5. (c) $s_{2}=0.8, \kappa=4, s_{1}=2$. (d) $s_{2}=-0.8, \kappa=-2, s_{1}=-1$. (e) $s_{2}=0.8, \kappa=-4, s_{1}=1$.

Regime D also exhibits a threshold $|\mu|$ value for soliton existence but in this regime the threshold is set by the CA solution criterion [Eq. (17)]. This threshold is therefore $\mu=\kappa / 2$.

It is clear that there are no areas where all three conditions overlap in regime $\mathrm{C}$ [shown in Fig. 1(c)]. In this regime the dispersion in the FF and $\mathrm{SH}$ have opposite signs. Previous work investigating this regime [19] has predicted the existence of quasisolitons where the FF component is localized but the $\mathrm{SH}$ has oscillating tails that do not decay far from the center of the pulse. The existence of these quasisolitons requires the localization in the FF and the CA solution to exist but does not require localization in the $\mathrm{SH}$. We therefore expect the existence of these quasisolitons for all $\mu<0$ in regime $\mathrm{C}$. It follows that similar quasisolitons should exist below the threshold in regime $\mathrm{B}$ where the condition for $\mathrm{SH}$ localization is not met (but the other two conditions are met). 
Finally regime E exists where $\kappa<0$ and opposite signs of dispersion in the FF and $\mathrm{SH}$. Although clearly similar to regime $\mathrm{C}$, in this regime a threshold for quasisoliton existence is predicted. Here for negative $\mu$, when $|\mu|<|\kappa / 2|$ no solitons or quasisolitons are expected to exist. However, for $|\mu|>|\kappa / 2|$ the condition for CA solutions is met but $\mathrm{SH}$ localization is not. Therefore we predict the existence of quasisolitons here.

We suggest the term "hard" threshold to describe a threshold set by the CA solution existence criterion (17), as in regimes D and E. Under this threshold no solitons or quasisolitons are predicted at all. The term "soft" threshold is suggested to describe a threshold set by the $\mathrm{SH}$ localization condition [Eq. (14)] as in regime B, where quasisolitons exist below the threshold.

\section{B. Interaction with dispersive waves}

In the previous section we have discussed the existence of solitons and quasisolitons under the condition of constant GVD [Eq. (9)]. Relaxing this condition to allow for the more general case of nonconstant GVD gives the opportunity for further analysis. Consider additional terms such that Eq. (9) becomes

$$
\begin{aligned}
& D_{f}(\delta)=-r_{2} \delta^{2}+C_{f}(\delta), \\
& D_{s}(\delta)=s_{1} \delta-s_{2} \delta^{2}+C_{s}(\delta),
\end{aligned}
$$

where $C_{f}(\delta)$ and $C_{s}(\delta)$ are corrections to the constant GVD of the form

$$
\begin{gathered}
C_{f}=-\sum_{j=3}^{\infty} r_{j} \delta^{j}, \\
C_{s}=-\sum_{j=3}^{\infty} s_{j} \delta^{j} .
\end{gathered}
$$

If these corrections are small the constant GVD soliton solutions should still be approximate solutions. Practically speaking these terms often become relevant close to a zero-GVD point. To analyze this, dispersive waves (DWs) are included into the solution as perturbations of the form

$$
\begin{aligned}
U_{f} & =\left[W_{f}(\eta)+a_{f}(\eta, \xi)\right] e^{i \mu \xi}, \\
U_{s} & =\left[W_{s}(\eta)+a_{s}(\eta, \xi)\right] e^{i(2 \mu-\kappa) \xi},
\end{aligned}
$$

where $a_{f}$ and $a_{s}$ are small perturbations to the solitons such that $\left|a_{f} / W_{f}\right| \ll 1$ and $\left|a_{s} / W_{s}\right| \ll 1$. Considering these perturbations as a linear combination of generated (resonant) and existing (pump) DWs gives

$$
\begin{aligned}
& a_{f}=\psi_{f}+p_{f} e^{i \phi_{f}\left(\delta_{f}\right)}, \\
& a_{s}=\psi_{s}+p_{s} e^{i \phi_{s}\left(\delta_{s}\right)},
\end{aligned}
$$

where $p_{f, s}$ is the real amplitude of the pump DWs and $\phi_{f}(\delta)=$ $q_{f}(\delta) \xi-\delta_{f} \eta$ and similarly for $\phi_{s}$. $\psi_{f}$ are the generated resonant DWs. In general $a_{f, s}, p_{f, s}$, and $\psi_{f, s}$ are all functions of $\eta$ and $\xi . \delta_{f, s}$ is the frequency detuning of the pump DWs in the FF and SH respectively and the dispersion in the reference frame of the soliton is

$$
\begin{aligned}
& q_{f}(\delta)=D_{f}(\delta)-\mu-v \delta, \\
& q_{s}(\delta)=D_{s}(\delta)-2 \mu+\kappa-\nu \delta .
\end{aligned}
$$

Substituting Eqs. (21) and (22) into Eq. (3) and taking the Fourier transform gives

$$
\begin{aligned}
{\left[i \partial_{\xi}+\right.} & \left.q_{f}(\delta)\right] \tilde{\psi}_{f}+\left[\tilde{W}_{s} * \tilde{\psi}_{f}^{*}+\tilde{W}_{f}^{*} * \tilde{\psi}_{s}\right] \\
= & C_{f}(\delta) \tilde{W}_{f}(\delta)-\tilde{W}_{s}(\delta) * \tilde{p}_{f}(\delta) e^{-i q_{f}\left(\delta_{f}\right) \xi} \\
& -\tilde{W}_{f}^{*}(-\delta) * \tilde{p}_{s}(\delta) e^{i q_{s}\left(\delta_{s}\right) \xi}, \\
{\left[i \partial_{\xi}\right.} & \left.+q_{s}(\delta)\right] \tilde{\psi}_{s}+\left[\tilde{W}_{f} * \tilde{\psi}_{f}\right] \\
\quad= & C_{s}(\delta) \tilde{W}_{s}-\tilde{W}_{f} * \tilde{p}_{f}(\delta) e^{i q_{f}\left(\delta_{f}\right) \xi},
\end{aligned}
$$

where $\tilde{f}(\delta)$ denotes the Fourier transform of any function $f(t)$, and $f(t) * g(t)$ denotes a convolution of functions $f(t)$ and $g(t)$. The left-hand side (LHS) acts as an oscillator driven by the terms on the right-hand side (RHS). As $\tilde{\psi}_{f, s}$ and $\tilde{W}_{s, f}$ are all localized functions, their convolution [second terms on LHS of Eqs. (24)] will only make small contributions to the resonant frequencies of the system. For this reason these terms are neglected in the following analysis.

\section{Cherenkov radiation}

In the case of a system initially free from dispersive waves $\left(p_{f}=p_{s}=0\right)$, Eq. (24) simplifies to the driven oscillator equations,

$$
\begin{aligned}
{\left[i \partial_{\xi}+q_{f}(\delta)\right] \tilde{\psi}_{f} } & =C_{f}(\delta) \tilde{W}_{f}, \\
{\left[i \partial_{\xi}+q_{s}(\delta)\right] \tilde{\psi}_{s} } & =C_{s}(\delta) \tilde{W}_{s} .
\end{aligned}
$$

Taking $\psi_{f}$ of the form $\psi_{f} \propto e^{i q_{f} \xi-i \delta \tau}$ (and similar for $\psi_{s}$ ) and matching wave numbers between the oscillating and driving terms gives the resonant conditions

$$
\begin{aligned}
& q_{f}(\delta)=0, \\
& q_{s}(\delta)=0 .
\end{aligned}
$$

Where these conditions hold true the soliton is resonant with dispersive waves in the system. Radiation with the wave vector $q_{f, s}$ (and corresponding frequency detuning $\delta$ ) satisfying these conditions will be emitted from the soliton. This process is known as resonant or Cherenkov radiation [6]. In the previous section solitons were found to be possible in regimes $\mathrm{A}, \mathrm{B}$, and D. With corrections to the constant GVD $\left[C_{f}(\delta)\right.$ and $\left.C_{s}(\delta)\right]$, these solitons become resonant with dispersive waves in the system and emit Cherenkov radiation. As they are no longer localized the solitons become quasisolitons.

Similar analysis can be applied to the quasisolitons in regimes $\mathrm{B}, \mathrm{C}$, and $\mathrm{E}$. We find that $\mathrm{SH}$ dispersion $\left(s_{1}\right.$ and $\left.s_{2}\right)$ acts as the perturbation in this case. With these terms set to zero a soliton solution exists. Reinstating these terms produces resonances between the soliton and dispersive waves in the system and quasisolitons are predicted. At these resonances Cherenkov radiation is expected, this can also be interpreted as the oscillating tails of the soliton. The addition of further dispersive terms $C_{f}(\delta)$ and $C_{S}(\delta)$ may shift the frequency of this Cherenkov radiation. 


\section{Pumped radiation}

Any waves in the system that are not part of the soliton may perturb the soliton leading to emission of DWs at new frequencies. Here they are referred to as pump DWs, $p_{f}$ and $p_{s}$, in the FF and $\mathrm{SH}$ respectively. This includes any deliberately introduced pump into the system or radiation previously emitted by the soliton in question or other solitons in the system. In the following analysis pump DWs are assumed to be continuous wave and are therefore $\delta$ functions in frequency. This allows their convolutions to be evaluated simply giving

$$
\begin{aligned}
{\left[i \partial_{\xi}+q_{f}(\delta)\right] \tilde{\psi}_{f}=} & -p_{f} \tilde{W}_{s}\left(-\delta_{f}\right) e^{-i q_{f}\left(\delta_{f}\right) \xi} \\
& -p_{s}\left[\tilde{W}_{f}\left(-\delta_{s}\right)\right]^{*} e^{i q_{s}\left(\delta_{s}\right) \xi}, \\
{\left[i \partial_{\xi}+q_{s}(\delta)\right] \tilde{\psi}_{s}=} & -p_{f} \tilde{W}_{f}\left(\delta_{f}\right) e^{i q_{f}\left(\delta_{f}\right) \xi},
\end{aligned}
$$

with the resonant conditions

$$
\begin{aligned}
& q_{f}(\delta)=-q_{f}\left(\delta_{f}\right), \\
& q_{f}(\delta)=q_{s}\left(\delta_{s}\right), \\
& q_{s}(\delta)=q_{f}\left(\delta_{f}\right) .
\end{aligned}
$$

This radiation is produced by the interaction of the soliton and the pump DWs, and will therefore be referred to as pumped radiation. For a pump DW with a small detuning from the FF central frequency two resonant conditions exist, one in the FF and one in the SH. A pump DW with a small detuning from the $\mathrm{SH}$ central frequency has one possible resonance in the FF

Consider the specific case of previously emitted Cherenkov radiation, where $q_{f}\left(\delta_{f}\right)=0$ or $q_{s}\left(\delta_{s}\right)=0$. Substitution into Eq. (28) shows that the Cherenkov conditions from Eq. (26) are reproduced. This shows that previously emitted Cherenkov radiation cannot produce new frequencies when interacting with the soliton it was emitted from. In general, Cherenkov radiation emitted by one soliton interacting with a different soliton (with different central frequency, $\mu$ or $v$ ) would produce new frequencies.

\section{SOLITONS IN NANOWAVEGUIDES}

\section{A. Waveguide simulation}

In the previous section we have seen that existence of soliton and quasisoliton solutions depends strongly on the waveguide parameters. In this section we present simulated data for two waveguide structures. These data are analyzed from the point of view of soliton and quasisoliton existence as discussed in the previous section. This section is intended to clearly show how the generic theory presented so far maps onto specific waveguide geometries that can be experimentally realized.

Figures 2(a)-2(c) present simulated data for a lithium niobate on insulator (LNOI) structure [11,20,21]. The structure of this waveguide is shown in the inset of Fig. 2(b) and consists of a ridge of lithium niobate on a silica substrate. Figure 2(a) shows the $n_{\text {eff }}$ data for simulated FF and $\mathrm{SH}$ modes in the structure. The insets show the transverse mode profiles of the chosen modes. A fundamental mode was chosen for the FF where a higher-order mode was selected for the $\mathrm{SH}$, this allows phase matching between the modes. Phase-matching occurs when both modes have the same propagation constant $\beta(\omega)$ and from Eq. (1), the same effective index $n_{\mathrm{eff}}$. The phase-mismatch parameter $\kappa$ is shown in Fig. 2(b) and is zero at phase matching. It is clear that in this waveguide, phase matching occurs at around $1550 \mathrm{~nm}$.

Another important waveguide parameter to consider is the group velocity mismatch parameter $s_{1}$. This is plotted in Fig. 2(b). In this waveguide it is clear that $s_{1}$ rapidly decreases as wavelength increases but does not reach zero for the wavelengths shown. Figure 2(c) shows the GVD parameters $\beta_{2}$ for both $\mathrm{FF}$ and $\mathrm{SH}$ modes. Both modes show slowly varying GVD and with no zero-GVD points, the GVD of both modes remains normal for the wavelength range shown. The regimes of soliton existence outlined in the previous chapter are indicated in Fig. 2(c) by shaded areas and labeled with their corresponding letters. This waveguide supports regimes $\mathrm{B}$ and $\mathrm{D}$ for a broad range of wavelengths. From this we would expect solitons to be possible in this waveguide structure but with soft or hard thresholds depending on the regime (determined by wavelength).

Simulated data for the hybrid waveguide structure are shown in Figs. 2(d)-2(f). This hybrid structure consists of a suspended LN core and a silica microfiber; the cross section is given as an inset in Fig. 2(e). More details about this structure can be found in previous work [14,15,22]. Figure 2(d) shows simulated $n_{\text {eff }}$ data for FF and $\mathrm{SH}$ modes in this waveguide. Insets are included showing the transverse mode profiles. The phase mismatch and group-velocity mismatch parameters are plotted in Fig. 2(e). The GVD parameters for both modes are plotted in Fig. 2(f).

Comparison with the data from the LNOI structure shows clear differences. The hybrid structure shows a broad wavelength range where both $\kappa$ and $s_{1}$ are low. The GVD parameter for the $\mathrm{SH}$ also changed much more rapidly in the hybrid structure, exhibiting a zero-GVD point near the center of the wavelength range shown here. This zero-GVD point results in a change of sign of the $\mathrm{SH}$ dispersion making regimes in which FF and SH have opposite signs of dispersion available. Again the soliton regimes in this structure are plotted as shaded areas in Fig. 2(f). This structure provides three broad wavelength ranges where regimes $\mathrm{A}, \mathrm{C}$, and E exist. Therefore we would expect to find solitons and quasisolitons without any threshold in regimes $\mathrm{A}$ and $\mathrm{C}$ respectively. Quasisolitons with a hard threshold are expected in regime E. These two waveguide structures are therefore expected to exhibit all five of the predicted soliton regimes.

\section{B. Pulse propagation}

Using the data for the example nanowaveguides, we can simulate the propagation of pulses in these structures using the split-step Fourier method. In these simulations dispersion has been taken as a Taylor expansion truncated to third order such that the corrections to constant GVD are $C_{f}(\delta)=-r_{3} \delta^{3}$ and $C_{s}=-s_{3} \delta^{3}$. This approximation of dispersion as a truncated Taylor expansion is accurate close to the pulse central frequencies and allows the effects of correction terms $C_{f}$ and $C_{s}$ to be demonstrated.

In this section we present cross-correlation frequencyresolved optical gating (XFROG) spectrograms of pulses 


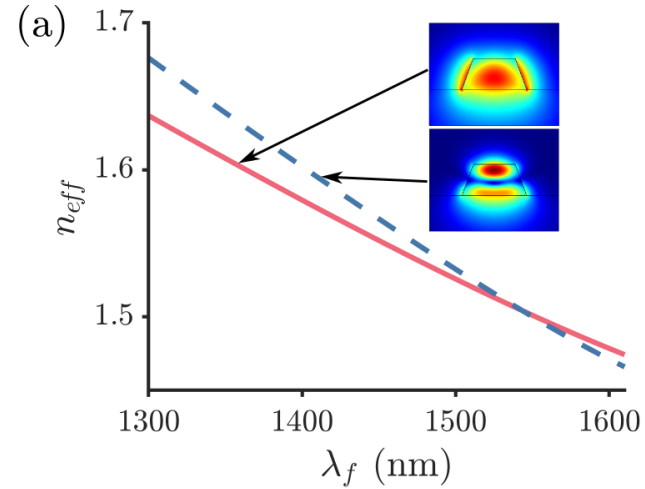

(b)
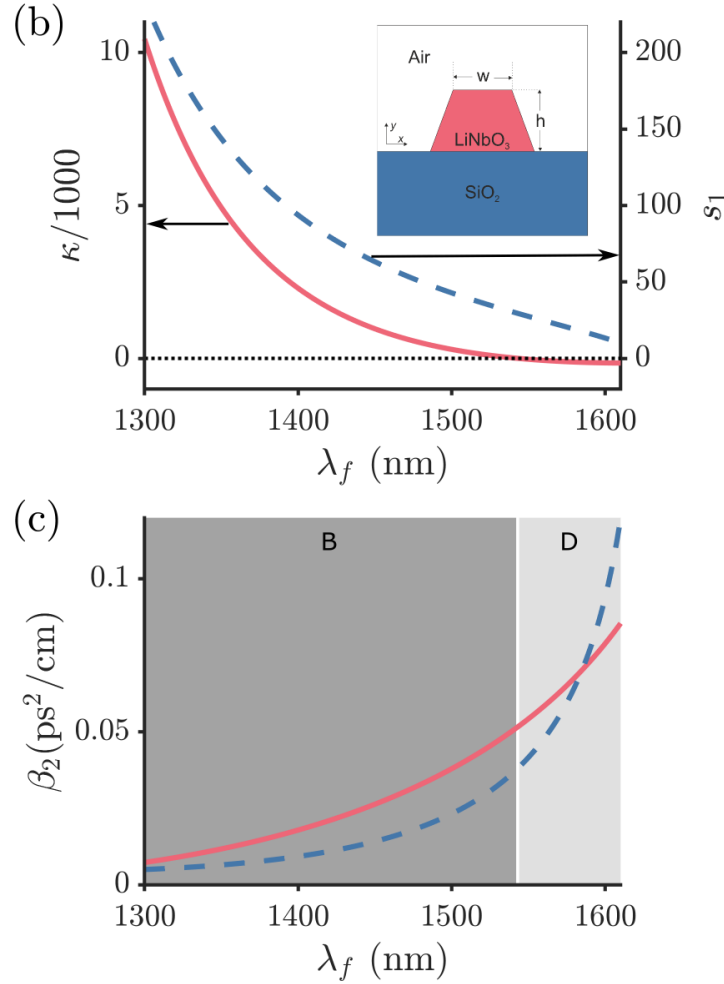

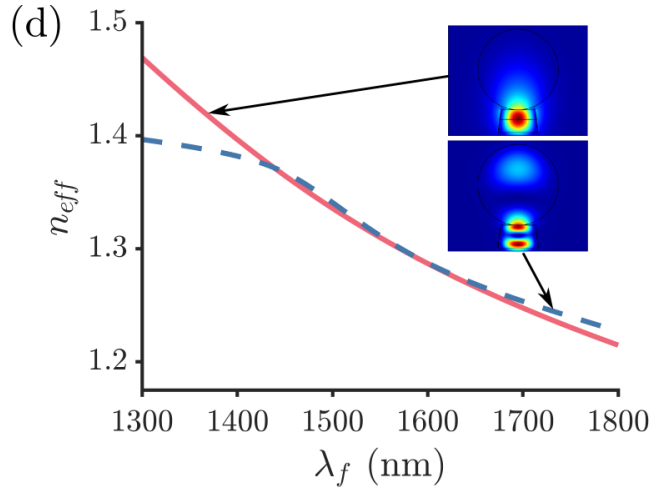

(e)
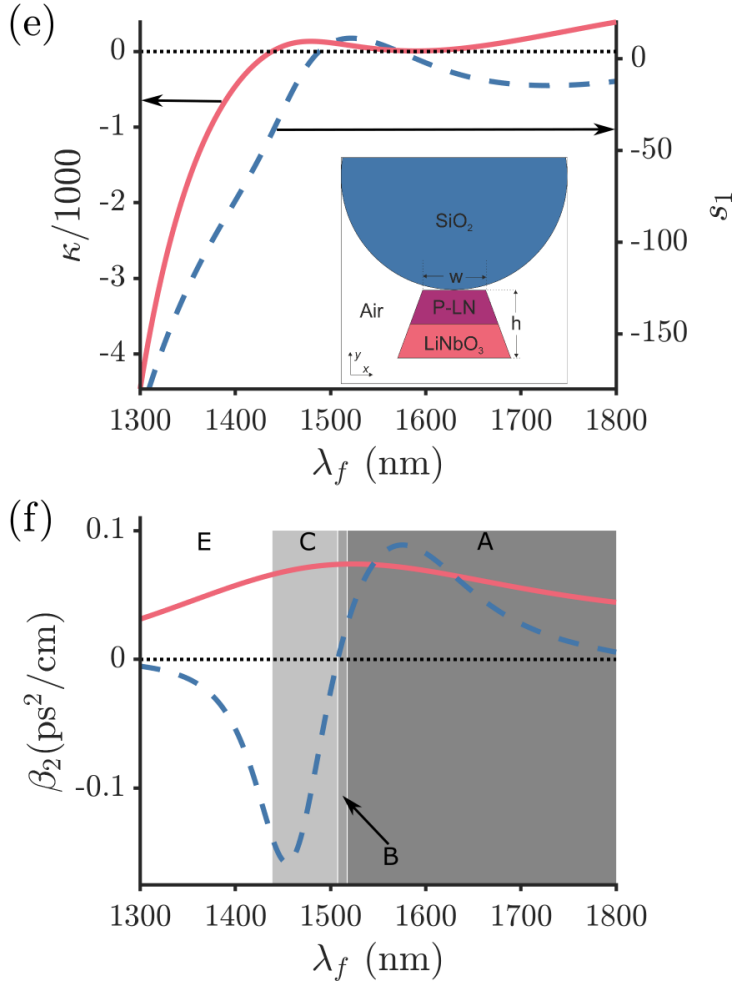

FIG. 2. For a lithium niobate on insulator (LNOI), $n_{\text {eff }}$ (a), $\kappa$ and $s_{1}$ (b), and $\beta_{2}$ (c) plotted against the FF wavelength $\lambda_{f}$. Insets in (a) show the FF (upper) and SH (lower) mode profiles. Inset in (b) shows the LNOI waveguide cross section, $h$ and $w$ label the height and width of the waveguide respectively. Data shown are for a LNOI structure with height of $350 \mathrm{~nm}$ and width of $500 \mathrm{~nm}$. For free-standing LN and microfiber (hybrid) structure, $n_{\text {eff }}(\mathrm{d}), \kappa$ and $s_{1}$ (e), and $\beta_{2}$ (f). Insets in (d) show the FF (upper) and SH (lower) mode profiles. Inset in (e) shows the cross section of the hybrid waveguide, $h$ and $w$ label the height and width of the waveguide respectively. Data shown are for a waveguide height of $300 \mathrm{~nm}$, width of $470 \mathrm{~nm}$, and a microfiber diameter of $1100 \mathrm{~nm}$. Solid and dashed curves (red and blue) are for FF and SH modes respectively. Dotted black lines mark $\kappa=0, s_{1}=0$, and $\beta_{2}=0$. Shaded regions mark the different soliton supporting regimes labeled A-E.

after propagation. An XFROG spectrogram is a well-known method for resolving both temporal and spectral features of a pulse $[5,6]$. Here the XFROGs were produced with the numerical integration of

$$
I(t, \omega)=\ln \left|\int_{-\infty}^{+\infty} d t^{\prime} A_{\mathrm{ref}}\left(t^{\prime}-t\right) U\left(t^{\prime}\right) e^{-i \omega t^{\prime}}\right|
$$

where $A_{\text {ref }}$ is the envelope of a Gaussian reference pulse, and $U$ is either the FF or SH field envelope. The plots for the analytic predictions of Cherenkov radiation are included for comparison using $\mu$ values estimated from the simulations. In all the simulations presented here, the emitted Cherenkov radiation matches closely with the resonance predictions.
In Fig. 3 the waveguides and wavelengths have been chosen such that each panel (a)-(e) represents each regime A-E. The input pulse for each simulation was set as in Eq. (12) for the FF field and zero in the SH field.

Figure 3(a) shows a soliton formed after propagation of a FF pulse in regime $\mathrm{A}$ in the hybrid structure. Although the input pulse used to generate the soliton here had a peak power of $1.1 \mathrm{~kW}$ we found soliton generation was possible for all peak powers attempted (lowest attempted was $15 \mathrm{~W}$ peak power). The resonance predictions in this figure show the dispersion truncated to both second order and third order. As expected for a localized soliton there are no resonances present when dispersion is truncated to second order. With third-order dispersion added there is a single resonance 
(a)
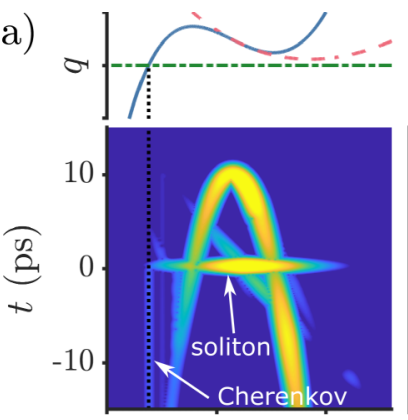

0.7

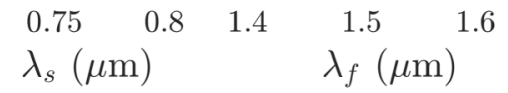

(b)
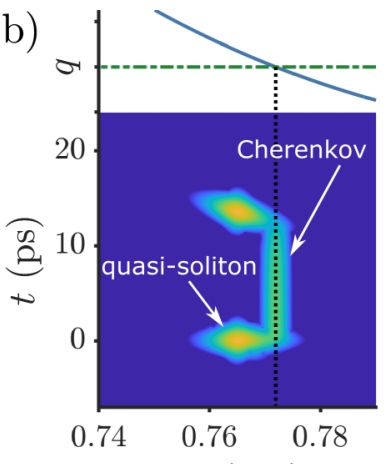

$\lambda_{s}(\mu \mathrm{m})$

(c)

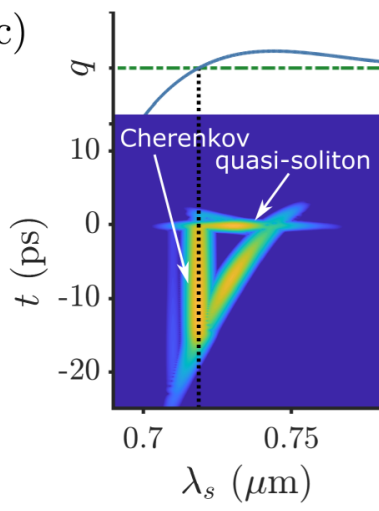

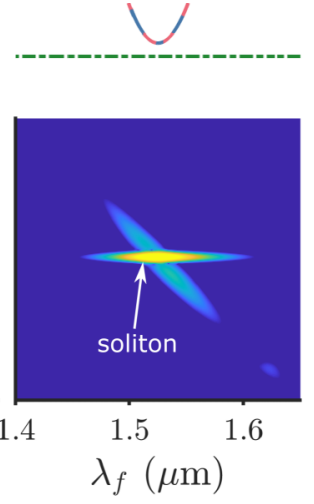
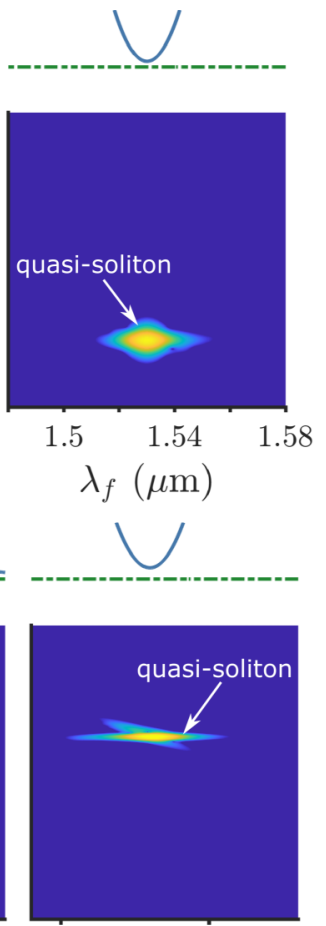

1.4

$\lambda_{f}(\mu \mathrm{m})$ (d)

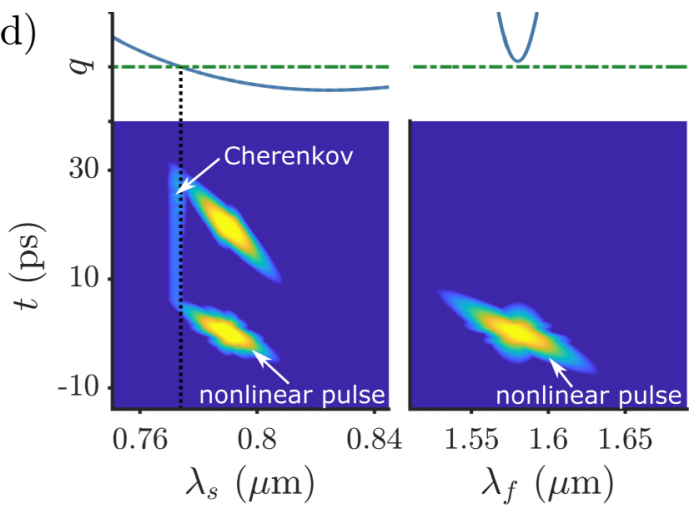

(e)
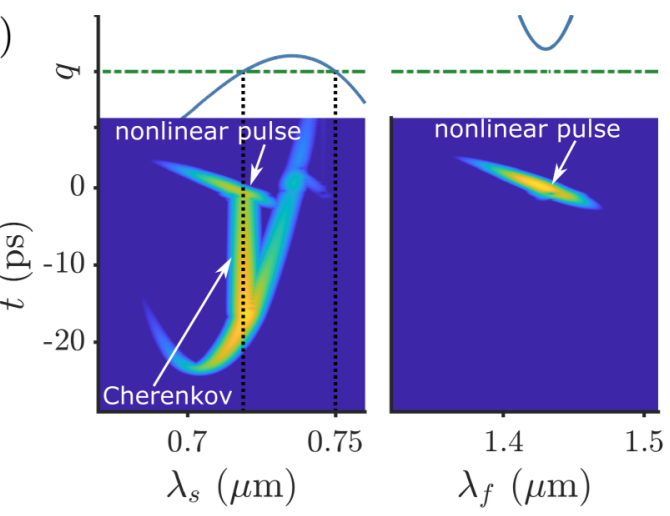

FIG. 3. XFROG plots of pulses after simulated propagation in nanowaveguide structures. Each panel consists of FF (right) and SH (left). Resonance predictions are included above XFROG plots for comparison. Wave vectors of dispersive waves are shown as solid lines (blue and red in color, truncated to third- and second-order dispersion respectively). Soliton wave vectors are shown as dot-dashed line (green). Resonances occur where these lines intersect and are marked by vertical dotted lines. (a), (c), and (e) are in the hybrid structure; (b) and (d) are in the LNOI structure. Input pulse parameters: (a) $1.1 \mathrm{~kW}$ peak power, 140 fs duration, central wavelength $1525 \mathrm{~nm}$, propagation distance $20 \mathrm{~mm}$. (b) $1.9 \mathrm{~kW}$ peak power, $560 \mathrm{fs}$ duration, central wavelength $1530 \mathrm{~nm}$, propagation distance $10 \mathrm{~mm}$. (c) $760 \mathrm{~W}$ peak power, $180 \mathrm{fs}$ duration, central wavelength $1460 \mathrm{~nm}$, propagation distance $10 \mathrm{~mm}$. (d) $14 \mathrm{~kW}$ peak power, $560 \mathrm{fs}$ duration, central wavelength $1580 \mathrm{~nm}$, propagation distance $30 \mathrm{~mm}$. (e) $1.2 \mathrm{~kW}$ peak power, $120 \mathrm{fs}$ duration, central wavelength $1430 \mathrm{~nm}$, propagation distance $10 \mathrm{~mm}$.

predicted far from the center of the soliton where lowintensity Cherenkov radiation is observed.

Figures 3(b) and 3(c) show quasisolitons formed in the LNOI and hybrid structures respectively. In both cases soliton component in the FF is localized but the $\mathrm{SH}$ is strongly emitting Cherenkov radiation. The wavelength of this radiation matches closely with that expected from the resonance predictions. This type of quasisoliton is predicted below the threshold in regime B and for all powers in regime C. At high powers solitons localized in both FF and SH are expected in regime $\mathrm{B}$ but due to the large walk-off $\left(s_{1}\right)$ in the LNOI structure this threshold is estimated to be $500 \mathrm{MW}$ peak power and therefore experimentally unattainable. Both these quasisolitons are expected to emit Cherenkov radiation without the inclusion of third-order dispersion which was verified. With the addition of third-order dispersion the wavelength of the resonances were shifted, and in the case of regime $\mathrm{C}$ one resonance was removed entirely leaving the quasisoliton shown in Fig. 3(c).

Figures 3(d) and 3(e) show pulses after propagation in regimes $\mathrm{D}$ and $\mathrm{E}$ respectively. Both of these regimes exhibit a hard threshold under which no solitons or quasisolitons are expected. In simulations in both of these regimes we were unable to find either solitons or quasisolitons at low 

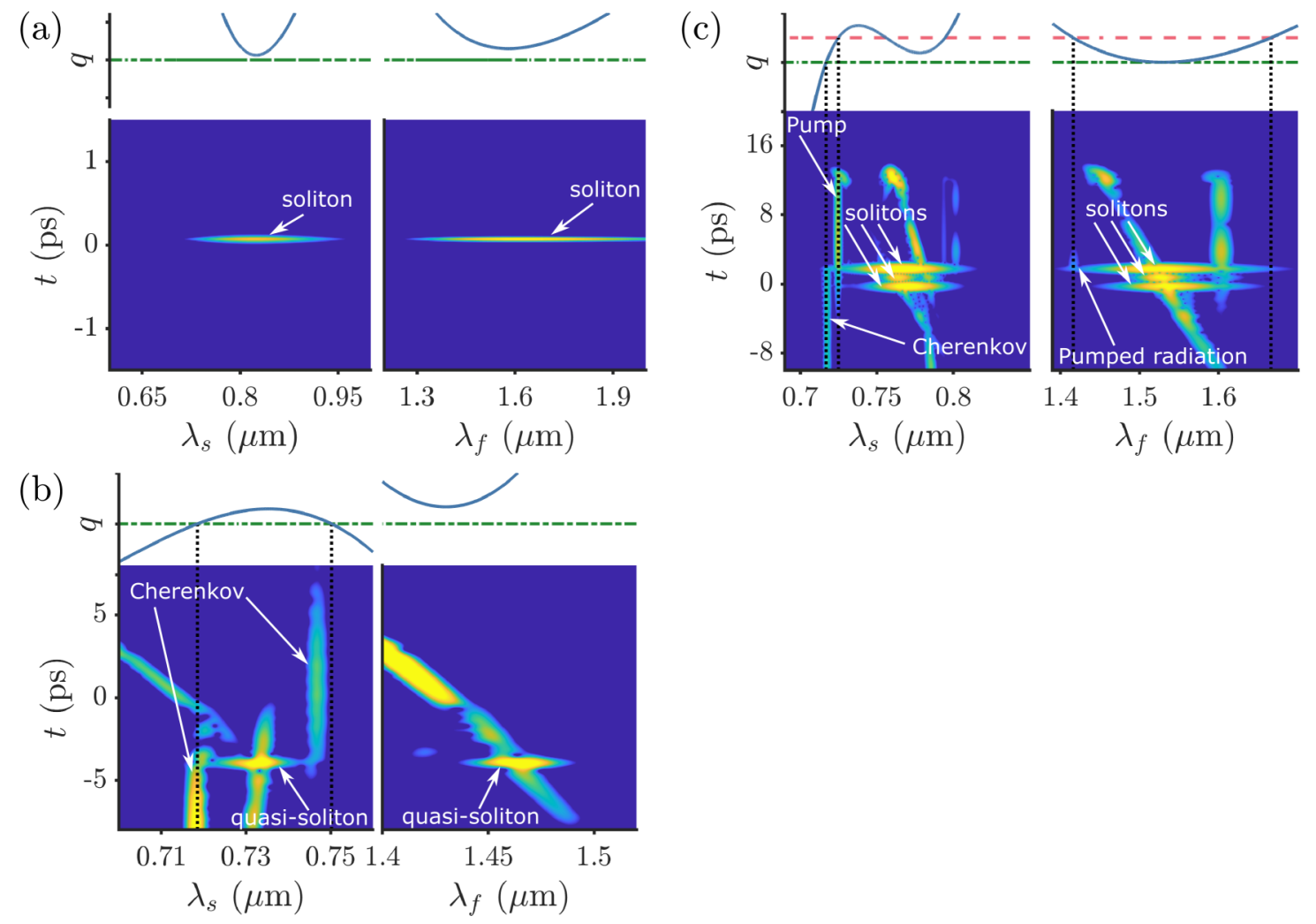

FIG. 4. XFROG plots of pulses after simulated propagation in nanowaveguide structures. Each panel consists of FF (right) and SH (left). Resonance predictions are included above XFROG plots for comparison. Wave vectors of dispersive waves are shown as blue solid lines (truncated to third-order dispersion). Soliton wave vectors are shown as green dot-dashed lines. Dashed red lines show the wave vectors of solitons interacting with pump frequencies. Resonances occur where these lines intersect. Vertical dotted lines mark the resonance wavelengths. (b) and (c) are in the hybrid structure; (a) is in the LNOI structure. Initial pulse parameters: (a) 6.3 and 19 MW peak power in the FF and SH respectively, $16 \mathrm{fs}$ duration, central wavelength $1580 \mathrm{~nm}$, propagation distance $10 \mathrm{~mm}$. (b) 17 and $21 \mathrm{~kW}$ peak power in the FF and SH respectively, $25 \mathrm{fs}$ duration, central wavelength $1430 \mathrm{~nm}$, propagation distance $15 \mathrm{~mm}$. (c) $2.7 \mathrm{~kW}$ peak power, $390 \mathrm{fs}$ duration, central wavelength $1530 \mathrm{~nm}$, propagation distance $26 \mathrm{~mm}$.

powers. Typically we found that a nonlinear pulse would form having components in the FF and SH. This pulse would exhibit some aspects of a soliton and quasisoliton such as Cherenkov radiation and the $\mathrm{SH}$ component staying locked to the FF component. These nonlinear pulses are, however, not solitons because they disperse as they propagate. Nonsolitonic pulses emitting resonant radiation have been predicted before in previous work $[23,24]$. In these regimes we did not observe formation of solitons at low powers even with the addition of $\mathrm{SH}$ pulses in the initial conditions.

Figure 4(a) shows a soliton after propagation in regime D. The initial conditions for this simulation was a numerically calculated soliton solution. This was necessary since the analytical solution [Eq. (12)] is no longer a good approximate solution in this regime. This result shows that for extremely high powers, solitons can exist in regime D. This high power is set by the hard threshold in this region.

It has not been possible to generate a quasisoliton in regime E. Attempts to excite quasisolitons in this regime have resulted in quasisolitons with a different central frequency, in a range that is not in regime E. An example of this type of soliton is shown in Fig. 4(b). We expect that exciting solitons in this regime is particularly difficult due to strong Cherenkov radiation expected from the quasisolitons in this regime destabilizing any potential solitons. The nearby existence of different regimes where soliton existence was more favorable is also expected to make soliton generation here more challenging.

Figure 4(c) shows the result after propagation of a broad higher-power pulse in regime A. The input field in the FF was of the form of the analytic soliton solutions [Eq. (12)] increased by a scale factor and zero in the SH. Multiple solitons are visible near $t=0$ with one of them emitting Cherenkov radiation. A soliton/pulse at around $t=12 \mathrm{ps}$ can be seen emitting its own Cherenkov radiation which is labeled "pump". This radiation interacts with one of the solitons near $t=0$, perturbing it and acting as a pump for further radiation. This further radiation can be seen labeled as "pumped radiation", and coincides with the resonance predictions. We can be sure that this pumped radiation is distinct from Cherenkov radiation because it begins to be formed as the soliton begins to interact with the pump waves. We find that the closely grouped solitons shown in Fig. 4(c) gradually separate and remain stable solitons after more than $100 \mathrm{~mm}$ of propagation.

Previous literature in this area suggests peak input powers of a few $\mathrm{kW}$ [20] are experimentally achievable for similar waveguides and wavelengths. This suggests that it should be experimentally possible to generate many of the solitons and 
quasisolitons observed here. It is important to highlight that the solitons predicted and simulated in Fig. 3(a) experience normal GVD $\left(\beta_{2}>0\right)$ in both the FF and SH. As Kerr solitons cannot exist for normal GVD [4] any experimentally observed solitons in this frequency range could only be due to $\chi^{(2)}$ nonlinearity.

\section{CONCLUSION}

The existence of temporal solitons in generic $\chi^{(2)}$ waveguides has been investigated. Conditions for the existence of localized solitons have been analyzed and five distinct regimes of soliton and quasisoliton existence have been identified. Two nanowaveguide structures were simulated and all five regimes were found to exist for different wavelengths in these two waveguides. Predictions for soliton and quasisoliton existence have been confirmed by pulse propagation simulation in the proposed nanowaveguide structures.

The interaction of solitons with higher-order dispersion terms has been predicted to produce Cherenkov radiation from both FF and $\mathrm{SH}$ soliton components. Soliton propagation simulations have confirmed these predictions. Radiation due to dispersive wave pumps has also been predicted and simulated. The wavelengths of this radiation in simulation coincides closely with those expected from analytic predictions. The generation of multiple solitons and quasisolitons under opposite signs of dispersion has also been simulated for experimentally attainable peak powers.

This model is intended to provide a useful theoretical basis for low-power soliton generation in $\chi^{(2)}$ waveguides. We hope that our analysis provides the possibility of optimizing soliton-assisted frequency conversion in lithium niobate nanowaveguides. Future work on the problem considered above can include analytical calculations of the amplitude of resonant radiation as has been done previously in Kerr systems $[6,25,26]$.

All data supporting this study are openly available from the University of Bath Research Data Archive [27].

\section{ACKNOWLEDGMENT}

W.R.R. acknowledges funding and support from the U.K. Engineering and Physical Sciences Research Council (EPSRC) Centre for Doctoral Training in Condensed Matter Physics (CDTCMP), Grant No. EP/L015544/1.
[1] Y. S. Kivshar and G. P. Agrawal, Optical Solitons: From Fibers to Photonic Crystals (Academic, New York, 2003), p. 540.

[2] G. P. Agrawal, Nonlinear Fiber Optics (Elsevier Science, New York, 2013).

[3] R. DeSalvo, H. Vanherzeele, D. J. Hagan, M. Sheik-Bahae, G. Stegeman, and E. W. Van Stryland, Opt. Lett. 17, 28 (1992).

[4] A. V. Buryak, P. D. Trapani, D. V. Skryabin, and S. Trillo, Phys. Rep. 370, 63 (2002).

[5] D. V. Skryabin and A. V. Gorbach, Rev. Mod. Phys. 82, 1287 (2010).

[6] D. V. Skryabin and A. V. Yulin, Phys. Rev. E 72, 016619 (2005).

[7] B. B. Zhou, X. Liu, H. R. Guo, X. L. Zeng, X. F. Chen, H. P. Chung, Y. H. Chen, and M. Bache, Phys. Rev. Lett. 118, 143901 (2017).

[8] M. Bache, O. Bang, B. B. Zhou, J. Moses, and F. W. Wise, Phys. Rev. A 82, 063806 (2010).

[9] B. Zhou and M. Bache, Opt. Lett. 40, 4257 (2015).

[10] J. Yang, B. A. Malomed, and D. J. Kaup, Phys. Rev. Lett. 83, 1958 (1999).

[11] G. Poberaj, H. Hu, W. Sohler, and P. Günter, Laser Photon. Rev. 6, 488 (2012).

[12] J. E. Toney, Lithium Niobate Photonics (Artech House, 2015).

[13] J.-Y. Chen, Y. M. Sua, H. Fan, and Y.-P. Huang, OSA Continuum 1, 229 (2018).

[14] A. Gorbach and W. Ding, Photonics 2, 946 (2015).

[15] L. Cai, A. V. Gorbach, Y. Wang, H. Hu, and W. Ding, Sci. Rep. 8, 12478 (2018).
[16] R. Wu, M. Wang, J. Xu, J. Qi, W. Chu, Z. Fang, J. Zhang, J. Zhou, L. Qiao, Z. Chai, J. Lin, and Y. Cheng, Nanomaterials 8, 910 (2018).

[17] Y. N. Karamzin and A.P. Sukhorukov, Sov. Phys.-JETP. 41, 414 (1975).

[18] S. Trillo and P. Ferro, Opt. Lett. 20, 438 (1995).

[19] K. Beckwitt, Y.-F. Chen, F. W. Wise, and B. A. Malomed, Phys. Rev. E 68, 057601 (2003).

[20] M. Yu, B. Desiatov, Y. Okawachi, A. L. Gaeta, and M. Lončar, Opt. Lett. 44, 1222 (2019).

[21] R. Luo, Y. He, H. Liang, M. Li, and Q. Lin, Optica 5, 1006 (2018).

[22] P. Main, P. J. Mosley, W. Ding, L. Zhang, and A. V. Gorbach, Phys. Rev. A 94, 063844 (2016).

[23] K. E. Webb, Y. Q. Xu, M. Erkintalo, and S. G. Murdoch, Opt. Lett. 38, 151 (2013).

[24] T. Roger, M. F. Saleh, S. Roy, F. Biancalana, C. Li, and D. Faccio, Phys. Rev. A 88, 051801(R) (2013).

[25] N. Akhmediev, A. Buryak, and M. Karlsson, Opt. Commun. 110, 540 (1994).

[26] N. Akhmediev and M. Karlsson, Phys. Rev. A 51, 2602 (1995).

[27] W. Rowe, D. Skryabin, and A. Gorbach, Data set for Temporal quadratic solitons and their interaction with dispersive waves in lithium niobate nanowaveguides, University of Bath Research Data Archive, https://doi.org/10.15125/BATH-00710. 